\title{
Widespread implementation of EUCAST breakpoints for antibacterial susceptibility testing in Europe
}

\author{
D Brown'1, R Cantón (rafael.canton@salud.madrid.org)2 , L Dubreuil3, S Gatermann4, C Giske5, A MacGowan', \\ L Martínez-Martínez ${ }^{7}$, J Mouton ${ }^{8}$, R Skov ${ }^{9}$, M Steinbakk $^{10}$, C Walton $^{11}$, O Heuer $^{12}$, M J Struelens ${ }^{12}$, L Diaz Högberg ${ }^{12}$, G Kahlmeter ${ }^{13}$ \\ 1. 222 Broadway, Peterborough, United Kingdom \\ 2. Hospital Universitario Ramón y Cajal and Instituto Ramón y Cajal de Investigación Sanitaria (IRYCIS), Madrid, Spain \\ 3. Faculté de Pharmacie, Lille, France \\ 4. Ruhr-Universität Bochum, Bochum, Germany \\ 5. Karolinska University Hospital, Stockholm, Sweden \\ 6. Southmead Hospital, Bristol, United Kingdom \\ 7. Hospital Universitario Marqués de Valdecilla, Santander, Spain \\ 8. Radboud University Nijmegen Medical Centre, Nijmegen, the Netherlands \\ 9. Statens Serum Institut, Copenhagen, Denmark \\ 10. Norwegian Institute of Public Health, Oslo, Norway \\ 11. External Quality Assurance Department, Public Health England, London, United Kingdom \\ 12. European Centre for Disease Prevention and Control (ECDC), Stockholm, Sweden \\ 13. Centrallasarettet, Växjö, Sweden
}

Citation style for this article:

Brown D, Cantón R, Dubreuil L, Gatermann S, Giske C, MacGowan A, Martínez-Martínez L, Mouton J, Skov R, Steinbakk M, Walton C, Heuer O, Struelens MJ, Diaz Högberg L, Kahlmeter G. Widespread implementation of EUCAST breakpoints for antibacterial susceptibility testing in Europe. Euro Surveill. 2015;20(2):pii=21008. Available online: http://www.eurosurveillance.org/ViewArticle.aspx?Articleld=21008

Article submitted on 31 January 2014 / published on 15 January 2015

The European Committee on Antimicrobial Susceptibility Testing (EUCAST) was established to harmonise clinical antimicrobial breakpoints and to define breakpoints for new agents in Europe. Data from the European Antimicrobial Resistance Surveillance Network (EARS-Net) external quality assessment (EQA) exercises from 2009 to 2012 , from the United Kingdom External Quality Assessment Scheme (UK NEQAS) from November 2009 to March 2013 and data collected by EUCAST through a questionnaire in the first quarter of 2013 were analysed to investigate implementation of EUCAST guidelines in Europe. A rapid change to use of EUCAST breakpoints was observed over time. Figures for implementation of EUCAST breakpoints at the end of the studied period were $61.2 \%$ from EARSNet data and $73.2 \%$ from UK NEQAS data. Responses to the EUCAST questionnaire indicated that EUCAST breakpoints were used byover $50 \%$ of laboratories in 18 countries, by 10 to $50 \%$ of laboratories in eight countries and by less than $10 \%$ in seven countries. The EUCAST disk diffusion method was used by more than $50 \%$ of laboratories in 12 countries, by 10 to $50 \%$ of laboratories in ten countries and byless than $10 \%$ in eleven countries. EUCAST guidelines implementation is essential to ensure consistent clinical reporting of antimicrobial susceptibility results and antimicrobial resistance surveillance.

\section{Background}

The use of common clinical breakpoints for antimicrobial susceptibility testing is important both for consistent clinical reporting of antimicrobial susceptibility and for international surveillance of the antimicrobial susceptibility of microorganisms. The principal objective of the European Committee on Antimicrobial Susceptibility Testing (EUCAST) [1] is to harmonise antimicrobial breakpoints in Europe and to define breakpoints for new agents in collaboration with the European Medicines Agency (EMA) [2] following a standard operating procedure agreed between EUCAST and the EMA [3,4]. EUCAST was established by the European Society for Clinical Microbiology and Infectious Diseases (ESCMID) in 1997 [5]. The committee was restructured in the years 2001 and 2002 with the support and central involvement of the national breakpoint committees that were active in Europe, i.e. those in France, Germany, the Netherlands, Norway, Sweden and the United Kingdom, and has been in operation in its current form since 2002. EUCAST has a General Committee [6], which includes one representative of each country from Europe and any country outside Europe interested in being part of the EUCAST process.

ESCMID has remained the administrative, financial and scientific platform of EUCAST throughout. Principal financial support over the years has been from ESCMID, European Union (EU) grants, a grant from the European Centre for Disease Prevention and Control (ECDC) and currently through a framework contract with the ECDC.

Today, EUCAST is well established as the only panEuropean antimicrobial breakpoint committee, with representatives throughout Europe and beyond. It is accepted as the European antimicrobial breakpoint committee by clinicians and clinical microbiologists, by 
national breakpoint committees and medicines agencies in Europe, the ECDC, the EMA, the European Food Safety Authority (EFSA), the pharmaceutical industry and diagnostic companies with interests in antimicrobial susceptibility testing. Of note, the EUCAST clinical breakpoints apply to antimicrobial resistance case definition as reportable to the European Union (EU) surveillance network for communicable diseases [7].

The breakpoint harmonisation process for all major groups of antimicrobial agents and organisms was completed in 2008/09. Since then there has been rapid adoption of EUCAST breakpoints and methods in Europe. Complete data on uptake in all European laboratories are not available as in most countries there is no mechanism for collection of information on susceptibility testing guidelines followed. A combination of different data sources needs to be used to obtain this information.

\section{Analysed data sources}

Data presented here are taken from three different sources. Firstly, the external quality assessment (EQA) exercise that is part of the European Antimicrobial Resistance Surveillance Network (EARS-Net) [8] organised by ECDC though a framework contract with the UK National External Quality Assessment Scheme (UK NEQAS). Secondly, the international external quality assessment scheme run by UK NEQAS [9]. Thirdly, data collected by EUCAST in the first quarter of 2013 through a questionnaire on guidelines and methods used in different countries.

\section{EARS-Net external quality assessment}

The ECDC EARS-Net resistance surveillance programme collects data from all EU countries, two European Economic Area countries (Norway and Iceland) [7], plus Bosnia, Croatia (also EU since 1 July 2014), Israel and Turkey between 2009 and 2011 only. The number of participating laboratories in each country varies, with a total of between 766 and 817 laboratories from 28 to 30 countries participating in the annual EQA exercises between 2009 and 2012 [10-13]. As part of the EQA exercise information is collected on breakpoint guidelines followed and methods used.

\section{UK NEQAS for antimicrobial susceptibility testing}

The UK NEQAS EQA scheme [9] includes subscribing laboratories principally from European countries and, as with EARS-Net, the number of participating laboratories in each country is variable. However, the distribution of numbers of laboratories among countries differs from that of EARS-Net, with a total of between 632 and 656 laboratories participating in the EQA scheme between November 2009 and March 2013. In the UK NEQAS for antimicrobial susceptibility testing two organisms of a variety of species are distributed each month. The number of participating laboratories returning results varies with the organism and antimicrobial agent so for consistency the data are based on results returned for $E$. coli isolates tested against ciprofloxacin, one of the most widely tested combinations. For each organism distributed, information is collected on breakpoint guidelines followed and methods used.

\section{EUCAST questionnaire on guidelines and methods used in different countries} In the first quarter of 2013, a questionnaire was distributed to all General Committee members with the objective of collecting information on whether EUCAST breakpoint guidelines were followed, adoption of the EUCAST disk diffusion method and whether the country has a national antimicrobial susceptibility committee (NAC) as recommended by EUCAST [14]. At that time there were 35 countries with national representatives on the EUCAST General Committee, Australia, Austria, Belgium, Bosnia and Herzegovina, Bulgaria, Croatia, Czech Republic, Denmark, Estonia, Finland, France, Germany, Greece, Hungary, Iceland, Ireland, Israel, Italy, Latvia, Lithuania, Luxemburg, the Netherlands, Norway, Poland, Portugal, Romania, Russia, Serbia, Slovakia, Slovenia, Spain, Sweden, Switzerland, Turkey and the United Kingdom. In most countries there is no official requirement for laboratories to follow any particular breakpoint guidelines and a variety of methods is used. Also most countries have no mechanism for collecting precise information on guidelines and methods used, and this may be continually changing as individual laboratories make decisions to change susceptibility testing guidelines followed or methods used. Therefore, the General Committee representatives were asked to provide estimates of the proportions of laboratories falling into broad categories for use of EUCAST guidelines and the EUCAST disk diffusion method. The categories provided were below $10 \%$, 10 to $50 \%$ and above $50 \%$ of laboratories.

\section{Results}

Data from EARS-Net (Table) show a decline in use of the Clinical and Laboratory Standards Institute (CLSI, United States) breakpoints from $67.5 \%$ in September 2009 to $38.4 \%$ in May 2012, and an increase in use of EUCAST breakpoints from $22.2 \%$ in 2009 to $61.2 \%$ in 2012. Some national guidelines such as the British Society for Antimicrobial Chemotherapy (BSAC, United Kingdom) [15] and the Comite de l'Antibiogramme de la Société Française de Microbiologie (CA-SFM, France) [16] have adopted EUCAST MIC breakpoints and initially calibrated their own disk diffusion method to the EUCAST breakpoints, so they were using EUCASTrelated methods and are therefore also counted as using EUCAST breakpoints. Both BSAC and CA-SFM are now in the process of changing to the EUCAST disk diffusion method.

Data from UK NEQAS EQA (Table) show a similar decline in use of CLSI breakpoints as seen in EARS-Net, from $58.5 \%$ in November 2009 to $26.8 \%$ in March 2013 and an increase in use of EUCAST breakpoints, from $36.1 \%$ to $73.2 \%$ over the same period. As with EARS-Net data, some national guidelines have adopted EUCAST 
Antimicrobial susceptibility testing guidelines used by laboratories participating in the EARS-Net EQA exercises, 2009-2012 and UK NEQAS for antimicrobial susceptibility testing, 2009-2013

\begin{tabular}{|c|c|c|c|c|}
\hline \multirow[b]{2}{*}{ Data source } & \multirow{2}{*}{$\begin{array}{l}\text { Date, } \\
\text { number of laboratories }\end{array}$} & \multicolumn{3}{|c|}{ Percentage of laboratories using indicated guidelines } \\
\hline & & CLSI & $\begin{array}{l}\text { EUCAST and } \\
\text { EUCAST-based }\end{array}$ & $\begin{array}{c}\text { Other }{ }^{\mathrm{a}} \\
\text { combined/ } \\
\text { not stated }\end{array}$ \\
\hline \multirow{4}{*}{ EARS-Net EQA } & $\begin{array}{c}\text { September } 2009 \\
n=775\end{array}$ & 67.5 & 22.2 & 10.3 \\
\hline & $\begin{array}{c}\text { June } 2010 \\
n=766\end{array}$ & 65.8 & 28.7 & $5 \cdot 5$ \\
\hline & $\begin{array}{c}\text { May } 2011 \\
\mathrm{n}=817\end{array}$ & 46.8 & 47.6 & 5.6 \\
\hline & $\begin{array}{c}\text { May } 2012 \\
\mathrm{n}=807\end{array}$ & 38.4 & 61.2 & 0.4 \\
\hline \multirow{5}{*}{ UK NEQAS EQA } & $\begin{array}{c}\text { November } 2009 \\
n=651\end{array}$ & 58.8 & 36.1 & 5.1 \\
\hline & $\begin{array}{c}\text { November } 2010 \\
n=656\end{array}$ & 51.5 & 42.2 & 6.3 \\
\hline & $\begin{array}{c}\text { November } 2011 \\
n=643\end{array}$ & 36.8 & 58.6 & 4.6 \\
\hline & $\begin{array}{c}\text { April } 2012 \\
\mathrm{n}=632\end{array}$ & 31.8 & 68.2 & 0 \\
\hline & $\begin{array}{c}\text { March } 2013 \\
\mathrm{n}=650\end{array}$ & 26.8 & 73.2 & 0 \\
\hline
\end{tabular}

CLSI: Clinical and Laboratory Standards Institute; EARS: European Antimicrobial Resistance Surveillance Network; EQA: external quality assessment; EUCAST: European Committee on Antimicrobial Susceptibility Testing; UK NEQAS: United Kingdom External Quality Assessment Scheme.

a Other guidelines are local methods not complying with EUCAST or CLSI recommendations.

MIC breakpoints and are therefore counted as using EUCAST breakpoints.

Questionnaires were completed by 33 of the 35 General Committee representatives. Countries with a NAC are shown in Figure 1. At the time of the survey, 25 of the responding countries had an established NAC, four were in the process of setting up a NAC and four had no NAC. Use of EUCAST breakpoint guidelines is shown in Figure 2. EUCAST breakpoints were used by more than $50 \%$ of laboratories in 18 countries, by 10 to $50 \%$ of laboratories in eight countries and by less than $10 \%$ in seven countries. Use of EUCAST disk diffusion method is shown in Figure 3. The EUCAST disk diffusion method was used by more than $50 \%$ of laboratories in 12 countries, by 10 to $50 \%$ of laboratories in ten countries and by less than $10 \%$ in eleven countries.

\section{Discussion}

Collection of reliable data on use of clinical breakpoint guidelines and methods for antimicrobial susceptibility testing in different countries is difficult because in most countries there is no national requirement to follow particular guidelines or methods, there are no mechanisms in place to collect such data, and the situation may change gradually over time as laboratories decide to change guidelines or methods. However, the findings from three independent data sources presented here consistently show that there has been widespread adoption of EUCAST breakpoints in recent years across clinical laboratories in the majority of European countries. The EQA exercises organised by EARS-Net and UK NEQAS include different but overlapping sets of laboratories covering most European countries. These EQA exercises show similar trends towards adoption of EUCAST breakpoints since 2009, with the UK NEQAS data indicating that over $70 \%$ of the laboratories providing data used EUCAST breakpoints in March 2013. The adoption of EUCAST guidelines has been mirrored by a decline in the use of CLSI breakpoints. This process has been fuelled by the adoption of EUCAST breakpoints by EMA in 2005 [3] as part of the official European process for marketing authorisation of antimicrobial agents, the adoption of EUCAST breakpoints by the European Commission Decision on case definition for surveillance of antimicrobial resistance in humans in 2012 [7], as well as the strong support by ESCMID and ECDC for use of EUCAST breakpoints for surveillance. Moreover, in some countries, the position taken by national societies of clinical microbiology and/or infectious diseases has had a positive impact.

The rate of adoption of EUCAST breakpoints has been variable in different countries, as illustrated by the results from the EUCAST survey early in 2013. While in just over half of the countries surveyed the majority of laboratories have adopted EUCAST breakpoints, in others the proportion of laboratories using EUCAST 


\section{FIGURE 1}

Countries with National Antimicrobial Susceptibility Testing Committees, EUCAST survey 2013

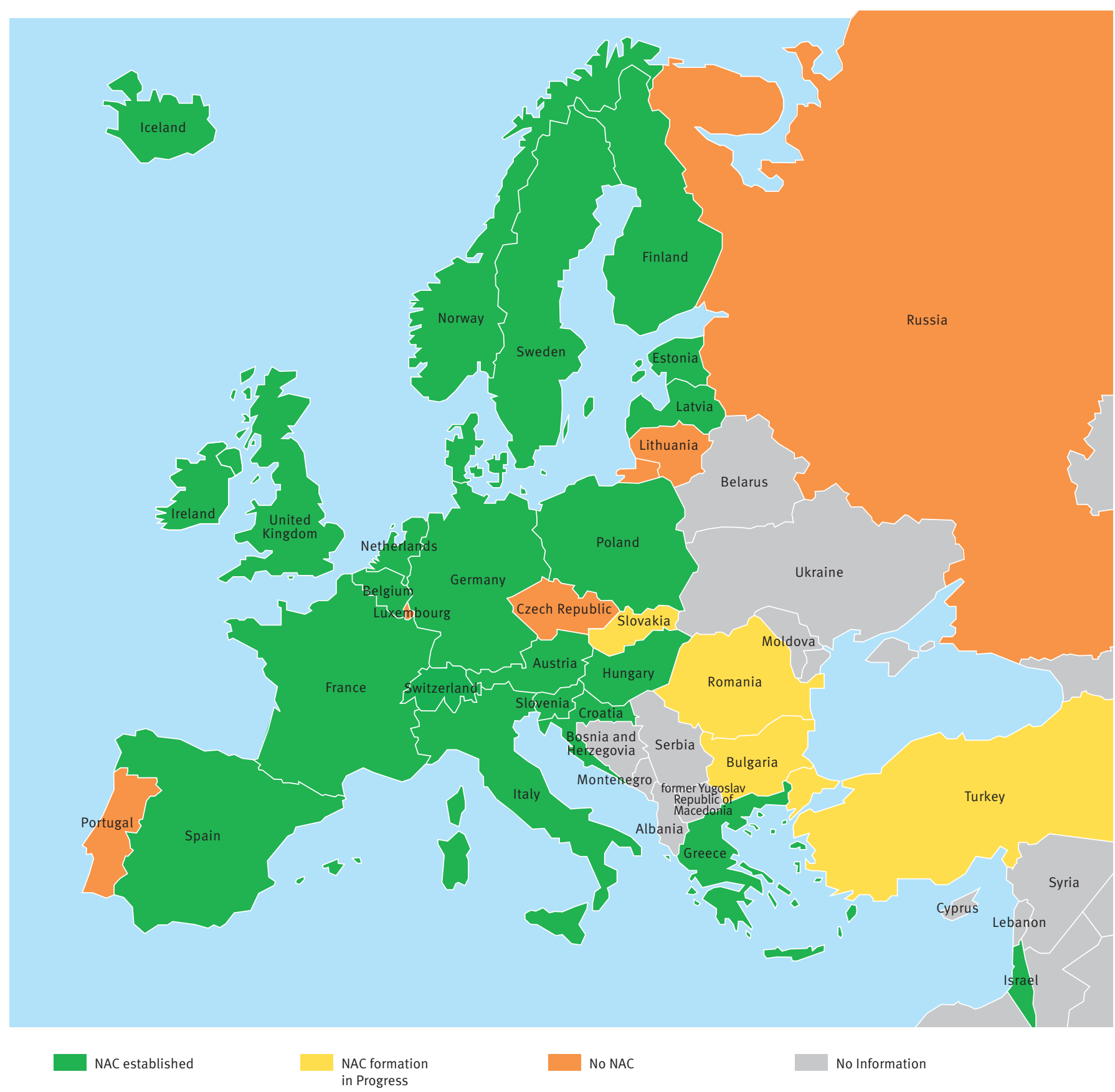

Australia (has NAC) is not on this map.

EUCAST: European Committee on Antimicrobial Susceptibility Testing, NAC: National Antimicrobial Susceptibility Testing Committee. 


\section{FIGURE 2}

Use of EUCAST breakpoint guidelines in different countries, EUCAST survey 2013

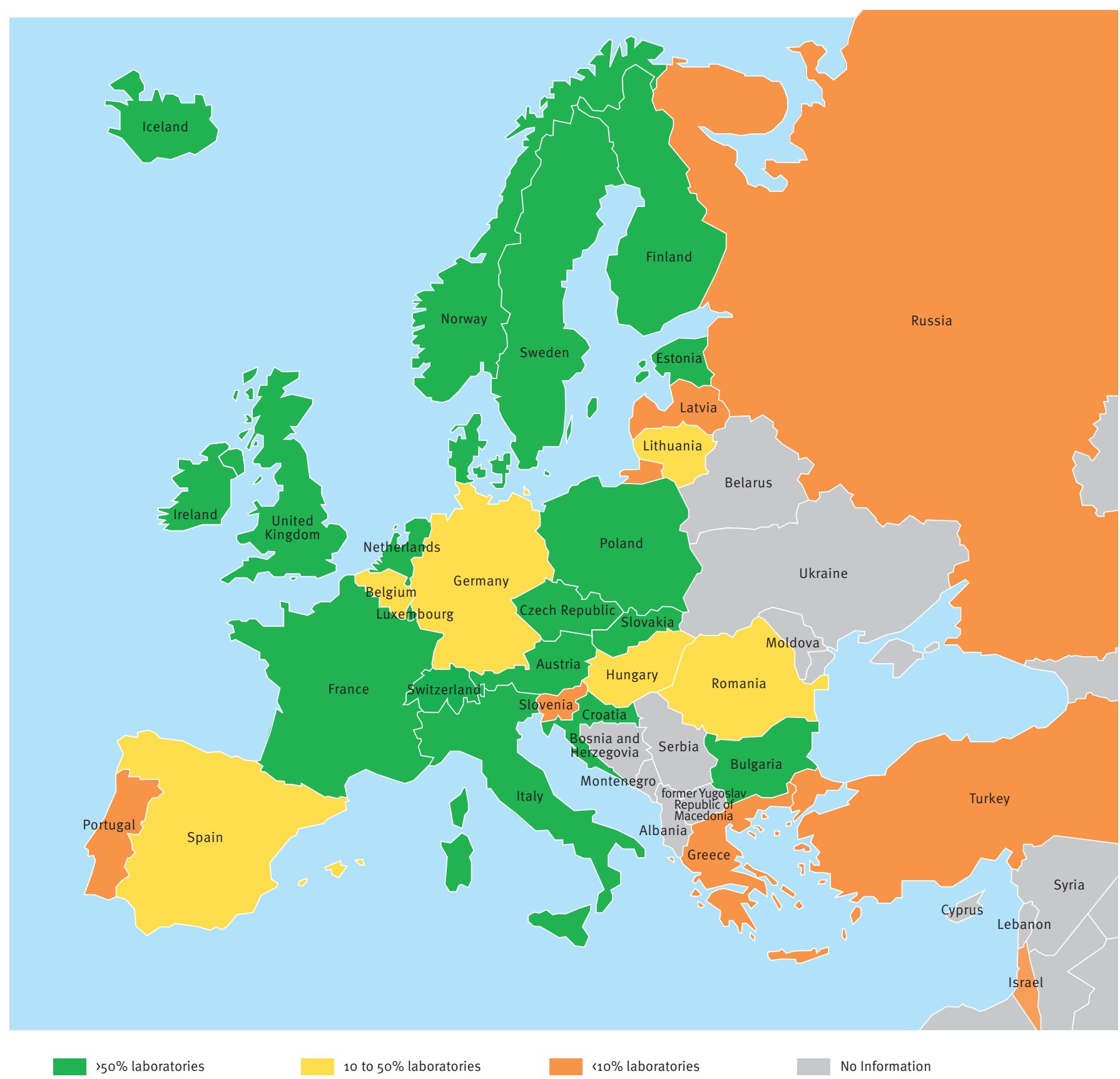

Australia (10 to $50 \%$ laboratories) is not on this map.

EUCAST: European Committee on Antimicrobial Susceptibility Testing. 


\section{FIGURE 3}

Use of the EUCAST disk diffusion method in different countries, EUCAST survey 2013

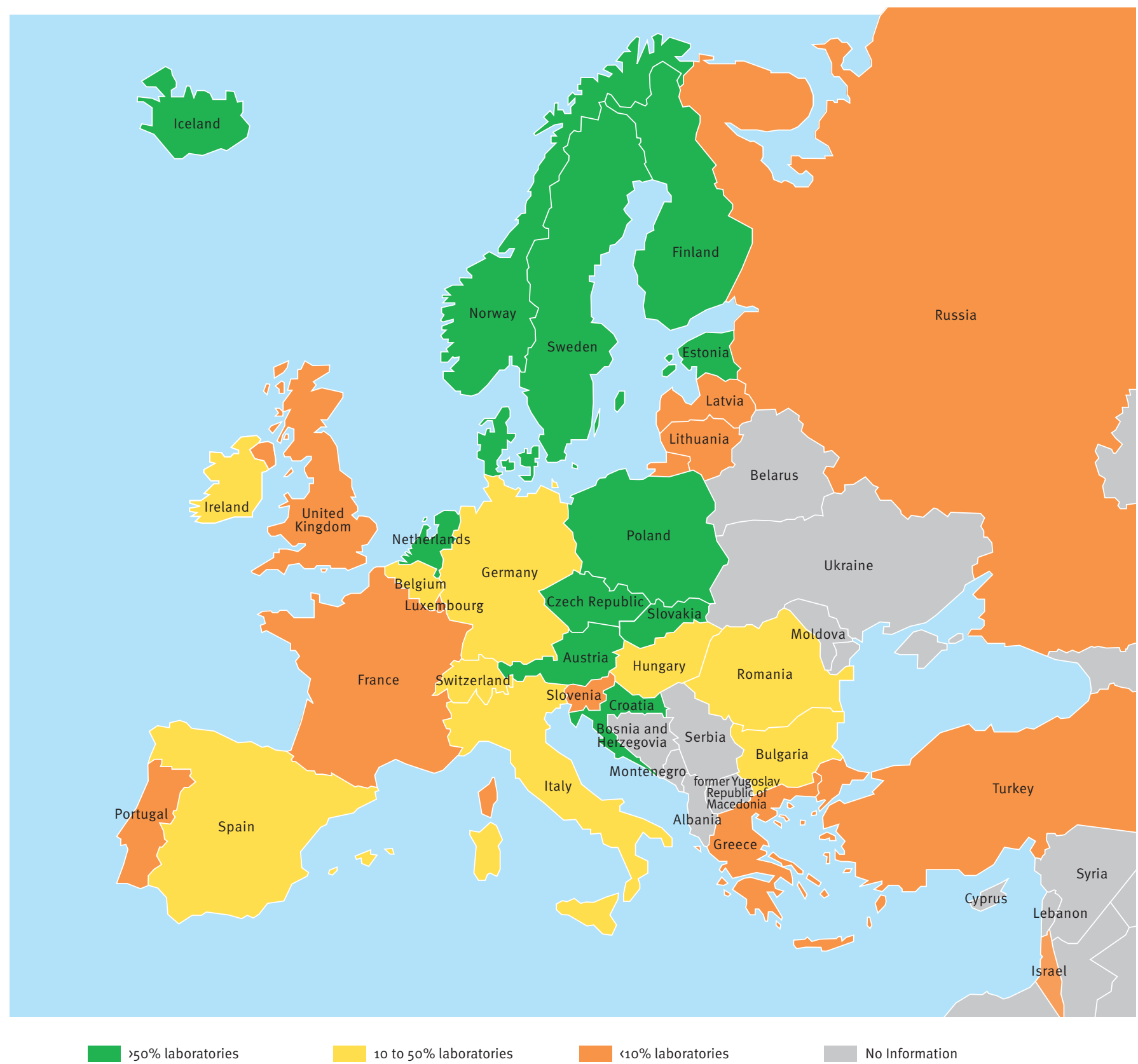

Australia (10 to $50 \%$ laboratories) is not on this map.

EUCAST: European Committee on Antimicrobial Susceptibility Testing. 
breakpoints is still small. It is expected that the uptake of guidelines will be gradual as laboratories make the decision to change breakpoints and incorporate breakpoints into local methods and information systems. The existence of a NAC to provide national guidance on antimicrobial susceptibility testing breakpoints and methods might have a substantial impact on laboratory practices. EUCAST has actively promoted the establishment of NACs in countries where no such group existed. The EUCAST survey shows that most countries now have a NAC or are in the process of setting up a NAC, and it is likely that these committees will positively influence the uptake of EUCAST guidelines. Furthermore, adoption of EUCAST breakpoints by public health microbiology national reference laboratories participating in ECDC-supported external quality assessment programmes will encourage alignment of testing practice across the EU. In addition, free access to EUCAST breakpoint documents via the internet and implementation of EUCAST breakpoints in automatic susceptibility testing devices facilitate the wide adoption of EUCAST guidelines.

Any standardised antimicrobial susceptibility testing method may be calibrated to EUCAST MIC breakpoints and national disk diffusion methods in France and the UK have been calibrated in this way $[17,18]$. However, there has been widespread demand for a EUCAST disk diffusion method and a EUCAST disk diffusion method was released in 2010 and published in 2014 [19]. The EUCAST 2013 survey has shown that, as with the uptake of EUCAST breakpoints, adoption of EUCAST disk diffusion method has been variable in different countries, but is used in a considerable proportion of laboratories in two thirds of surveyed countries. In many laboratories, the main antimicrobial susceptibility testing method is an automated system and delays in the implementation of EUCAST breakpoints in automated systems have delayed adoption of EUCAST breakpoints in some laboratories. However, the majority of EUCAST breakpoints are now implemented in automated systems [20] and laboratories can choose to use EUCAST breakpoints in their automated systems.

The information on uptake of EUCAST guidelines from EARS-Net and EUCAST relates only to clinical laboratories and the UK NEQAS EQA scheme includes greater than $95 \%$ of clinical laboratories. Information on guidelines followed in veterinary and food safety laboratories has not been surveyed by EUCAST but it would be useful to do so in collaboration with veterinary and food safety networks.

It is clear that there has been a rapid change to use of EUCAST breakpoints over the last few years and there are indications that this trend is continuing as EUCAST breakpoints are increasingly referred to in scientific communications. The wide adoption of EUCAST breakpoints will result in increased consistency of reporting of antimicrobial susceptibility testing results in different countries and better comparability of antimicrobial resistance surveillance data among countries. Annual monitoring of progress in implementation of EUCAST breakpoints across clinical and reference laboratories in Europe will be conducted jointly by EUCAST and ECDC as a key public health microbiology performance indicator.

\section{Acknowledgments}

We are grateful to the participants in ECDC EARS-Net and UK NEQAS who provided data in the respective EQA schemes; and to the EUCAST General Committee members who provided data relating to antimicrobial susceptibility testing in their respective countries.

\section{Conflicts of interest}

Derek Brown is Scientific Secretary of EUCAST, chairs the UK NEQAS Specialist Advisory Group on Antimicrobial Susceptibility Testing and advises UK NEQAS on Antimicrobial Susceptibility Testing issues. Rafael Cantón is Chairman of EUCAST and Gunnar Kahlmeter is Clinical Data Coordinator of EUCAST. Derek Brown, Gunnar Kahlmeter, Luc Dubreuil, Sören Gatermann, Christian Giske, Alasdair MacGowan, Luis Martínez-Martínez, Johan Mouton, Robert Skov, Martin Steinbakk and Rafael Cantón are members of the EUCAST Steering Committee. Christine Walton is the organiser of the UK NEQAS for Antimicrobial Susceptibility Testing. Ole Heuer and Liselotte Diaz Högberg administer the ECDC EARS-Net programme and Marc Struelens managed the ECDC service contract with EUCAST.

\section{Authors' contributions}

Derek Brown, Rafael Cantón and Gunnar Kahlmeter led the preparation of this manuscript. All members of the EUCAST Steering Committee were involved in the EUCAST survey and reviewed the manuscript. Christine Walton led the UK NEQAS team that organised and, with Derek Brown, analysed the data from the UK NEQAS and EARS-Net EQA distributions and also reviewed this manuscript. Ole Heuer, Liselotte Diaz Högberg and Marc Struelens reviewed and contributed to this manuscript.

\section{References}

1. European Committee on Antimicrobial Susceptibility Testing (EUCAST). [Internet]. [Accessed 1 Nov 2013]. Available from: http://www.eucast.org

2. European Medicines Agency (EMA). [Internet]. [Accessed 1 Nov 2013]. Available from: http://www.ema.europa.eu/ema

3. European Medicines Agency (EMA). Harmonisation of European Antimicrobial Susceptibility Testing Breakpoints determined by EMEA/CHMP and EUCAST SOP/H/3043. London: EMA. 23 Jan 2007. Available from: http://www.eucast.org/fileadmin/ $\mathrm{src} / \mathrm{media} / \mathrm{PDFs} / 4$ ESCMID_Library/3Publications/EUCAST Documents/Other_Documents/EMEA_CHMP_EUCAST_SOP_on Harmonising_European_Breakpoints_2007.pdf

4. European Committee on Antimicrobial Susceptibility Testing (EUCAST). Setting breakpoints for new antimicrobial agents, EUCAST SOP 1.1, 2013. Växjö: EUCAST. 1 Jun 2013. Available from: http://www.eucast.org/fileadmin/src/media/PDFs/ EUCAST_files/EUCAST_SOPs/EUCAST_SOP_1._1_Setting_ breakpoints_new_agents_1_June_2013.pdf

5. Kahlmeter G, Brown DF, Goldstein FW, MacGowan AP, Mouton JW, Osterlund A, et al. European harmonization of MIC breakpoints for antimicrobial susceptibility testing of bacteria. J Antimicrob Chemother. 2003;52(2):145-8. http://dx.doi. org/10.1093/jac/dkg312

6. European Committee on Antimicrobial Susceptibility Testing (EUCAST). General Committee. Växjö: EUCAST. [Accessed 
4 May 2014]. Available from: http://www.eucast.org/ organization/general_committee.

7. European Commission. Commission Implementing Decision of 8 August 2012 amending Decision 2002/253/EC laying down case definitions for reporting communicable diseases to the Community network under Decision No 2119/98/EC of the European Parliament and of the Council. Official Journal of the European Union. 2012;L262:1-2.

8. European Centre for Disease Prevention and Control (ECDC). European Antimicrobial Resistance Surveillance Network (EARS-Net). Stockholm: ECDC. [Accessed 4 May 2014]. Available from: http://www.ecdc.europa.eu/en/healthtopics/ antimicrobial_resistance/database/Pages/database.aspx

9. United Kingdom National External Quality Assessment Scheme for Microbiology (UK NEQAS). [Internet]. [Accessed 4 May 2014]. Available from: http://ukneqasmicro.org.uk/

10. European Centre for Disease Prevention and Control (ECDC). Antimicrobial Resistance Surveillance in Europe. Annual Report of the Antimicrobial Resistance Surveillance Network (EARSNet) 2009. Stockholm: ECDC; 2010. Available from: http:// www.ecdc.europa.eu/en/publications/Publications/1011_SUR_ annual_EARS_Net_2009.pdf

11. European Centre for Disease Prevention and Control (ECDC). Antimicrobial Resistance Surveillance in Europe. Annual Report of the Antimicrobial Resistance Surveillance Network (EARSNet) 2010. Stockholm: ECDC; 2011. Available from: http://www. ecdc.europa.eu/en/publications/Publications/1111_SUR_AMR_ data.pdf

12. European Centre for Disease Prevention and Control (ECDC). Antimicrobial Resistance Surveillance in Europe. Annual Report of the Antimicrobial Resistance Surveillance Network (EARSNet) 2011. Stockholm: ECDC; 2012. Available from: http://www. ecdc.europa.eu/en/publications/Publications/antimicrobialresistance-surveillance-europe-2011.pdf

13. European Centre for Disease Prevention and Control (ECDC). Antimicrobial Resistance Surveillance in Europe. Annual Report of the Antimicrobial Resistance Surveillance Network (EARSNet) 2012. Stockholm: ECDC; 2013. Available from: http://www. ecdc.europa.eu/en/publications/Publications/antimicrobialresistance-surveillance-europe-2012.pdf

14. European Committee on Antimicrobial Susceptibility Testing (EUCAST). National Antimicrobial Susceptibility Testing Committees. Växjö: EUCAST. [Accessed 4 May 2014]. Available from: http://www.eucast.org/organization/nac

15. British Society for Antimicrobial Chemotherapy (BSAC). Birmingham: BSAC. [Accessed 4 May 2014]. Available from: http://www.bsac.org.uk

16. Members of the SFM Antibiogram Committee. Comité de l'Antibiogramme de la Société Française de Microbiologie report 2003. Int J Antimicrob Agents. 2003;21(4):364-91. PMID:12672587

17. Howe RA, Andrews JM; BSAC Working Party on Susceptibility Testing. BSAC standardized disc susceptibility testing method (version 11). J Antimicrob Chemother. 2012;67(12):2783-4. http://dx.doi.org/10.1093/jac/dks391

18. Comité de l'antibiogramme de la Société Française de Microbiologie (CA SFM). Recommandations 2014 [2014 Recommendations]. [Accessed 6 Jan 2015]. French. Available from: http://www.sfm-microbiologie.org/UserFiles/files/ casfm/CASFM_EUCAST_V1_0_2014(1).pdf

19. Matuschek E, Brown DF, Kahlmeter G. Development of the EUCAST disk diffusion antimicrobial susceptibility testing method and its implementation in routine microbiology laboratories. Clin Microbiol Infect. 2014; 20(4):0255-66. http:// dx.doi.org/10.1111/1469-0691.12373

20. European Committee on Antimicrobial Susceptibility Testing (EUCAST). Compliance of manufacturers of AST materials and devices with EUCAST guidelines. Växjö: EUCAST. [Accessed 4 May 2014]. Available from: http://www.eucast.org/fileadmin/

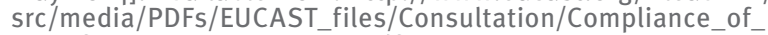
Manufacturers_2013-09-09.pdf 